\title{
Fascia Lata Donor Site Foreign Body Granuloma: An Unusual Presentation of a Rare Mimic of Soft Tissue Sarcoma
}

\author{
Olakunle F. Babalola ${ }^{1,2}$, Adedayo I. Salawu ${ }^{1,2}$, Abiodun I. Okunlola ${ }^{1,2}$, Oladipo Omoseebi ${ }^{3,4}$ \\ ${ }^{1}$ Department of Surgery, Federal Teaching Hospital, Ido-Ekiti, Nigeria \\ ${ }^{2}$ Department of Surgery, College of Medicine and Health Sciences, Afe Babalola University, Ado-Ekiti, Nigeria \\ ${ }^{3}$ Department of Morbid Anatomy, Federal Teaching Hospital, Ido-Ekiti, Nigeria \\ ${ }^{4}$ Department of Anatomic Pathology, College of Medicine and Health Sciences, Afe Babalola University, Ado-Ekiti, Nigeria \\ Email: *olakunlebabs@yahoo.com
}

How to cite this paper: Babalola, O.F., Salawu, A.I., Okunlola, A.I. and Omoseebi, O. (2021) Fascia Lata Donor Site Foreign Body Granuloma: An Unusual Presentation of a Rare Mimic of Soft Tissue Sarcoma. Surgical Science, 12, 31-36.

https://doi.org/10.4236/ss.2021.123005

Received: January 7, 2021

Accepted: March 2, 2021

Published: March 5, 2021

Copyright $\odot 2021$ by author(s) and Scientific Research Publishing Inc. This work is licensed under the Creative Commons Attribution International License (CC BY 4.0).

http://creativecommons.org/licenses/by/4.0/

\begin{abstract}
Background: Foreign body granuloma is a rare late complication of fascia lata graft donor site with few reported cases in the literature. It can mimic soft tissue sarcoma. Clinical and radiological findings may not be enough to solve the puzzle and histology remains the mainstay of diagnosis. Aim: Our aim is to highlight the unusual long interval between initial surgery and presentation of foreign body granuloma. Case Presentation: A 65-year-old man who presented 48 years after initial surgery with progressive painless fascia lata donor site soft tissue swelling. The management and histology findings were highlighted. Conclusion: The occurrence of foreign body granuloma remains an important differential following a history of past surgical procedure regardless of the time interval between the surgical procedure and the clinical presentation.
\end{abstract}

\section{Keywords}

Donor Site, Fascia Lata Graft, Foreign Body Granuloma, Soft Tissue Sarcoma

\section{Introduction}

The lateral thigh fascia lata is a common source of autologous tissue for many surgical procedures including Arthroplasty, Duroplasty, Heart valve replacement and Sling operations for Ptosis correction [1]. Most complications encountered at the donor site are usually transient, early and inconsequential. However few disabling complications have been reported such as muscle prolapse, reduced 
quadriceps muscle power and slightly limited hip range of motion [2] [3]. Insidious appearance of a progressively increasing tumour of the donor site decades after fascia lata harvest excites particular interest in view of the clinical significance of such a rare complication. Foreign body granulomas occurring at a site of previous surgical procedure or treatment have been termed gossypiboma or gauzeoma and are commonly found in the abdominal cavity [4]. We present a case report of a foreign body granuloma of an extremity fascia lata donor site mimicking soft tissue sarcoma with attendant diagnostic dilemma.

\section{Case Presentation}

A 65 year old man, retired media practitioner, presented to our clinic with 5-year history of painless progressively increasing swelling of the right lateral thigh, site of a previous fascia lata harvest for interpositional arthroplasty for post traumatic right elbow ankylosis 48 years prior to presentation. He had sustained a right elbow injury at the age of 17 -years while playing football in his college days. Five years prior to presentation, he suddenly noticed a swelling under the distal end of the lateral thigh scar, initially the size of his distal phalanx but later grew to the size of a tennis ball. There were no other body swellings or associated symptoms. Neither limitation of movement nor ulceration of the overlying skin occurred. Pertinent examination findings were in the extremities. There was a $12 \mathrm{~cm}$ well healed linear scar over the mid extensor surface of the right elbow with extension of the joint limited to 150 degrees. There was also a $10 \mathrm{~cm}$ flat, linear scar on the distal half of the right lateral thigh with a non tender, soft subcutaneous bulbous swelling extending from the proximal third of the scar to the distal end where it assumed a more rounded configuration and became fluctuant [Figure 1(a)]. There were neither differential temperature changes nor attachment to underlying muscle. A provisional diagnosis of soft tissue sarcoma was made. Haematological and electrolyte profile were normal. Plain radiograph of the right thigh revealed no bony involvement or any incidental radiopaque object. CT scan and MRI studies were not done due to financial issues as all payments were out of pocket with no insurance cover. Patient was adequately worked up for surgical intervention and had exploration of the lateral thigh subcutaneous space, complete gross excision of the well circumscribed and encapsulated mass overlying the whole length of the iliotibial tract, containing myxomatous, gelatinous substances, and measuring 20 by $8 \mathrm{~cm}$ in its widest dimension. Split thickness skin graft of the residual cutaneous defect [Figure 1(b)] following resection of the bulbous cystic degenerating mass attached to the overlying skin of the lower end was also done. Post operative period was uneventful and was discharged home on the $12^{\text {th }}$ post operative day. He was followed up for 3 years with no evidence of recurrence. Histopathological examination of the excised mass was reported as follows.

Microscopy skin tissues show papillomatous hyperplasia, hyperkeratosis and ulceration. Deep in the dermis are several large well formed granulomas adjacent to pseudo cavities containing inspissated secretions. The granulomas are replete with foreign body type multi-nucleated giant cells [Figure 2(a) and Figure 2(b)]. 

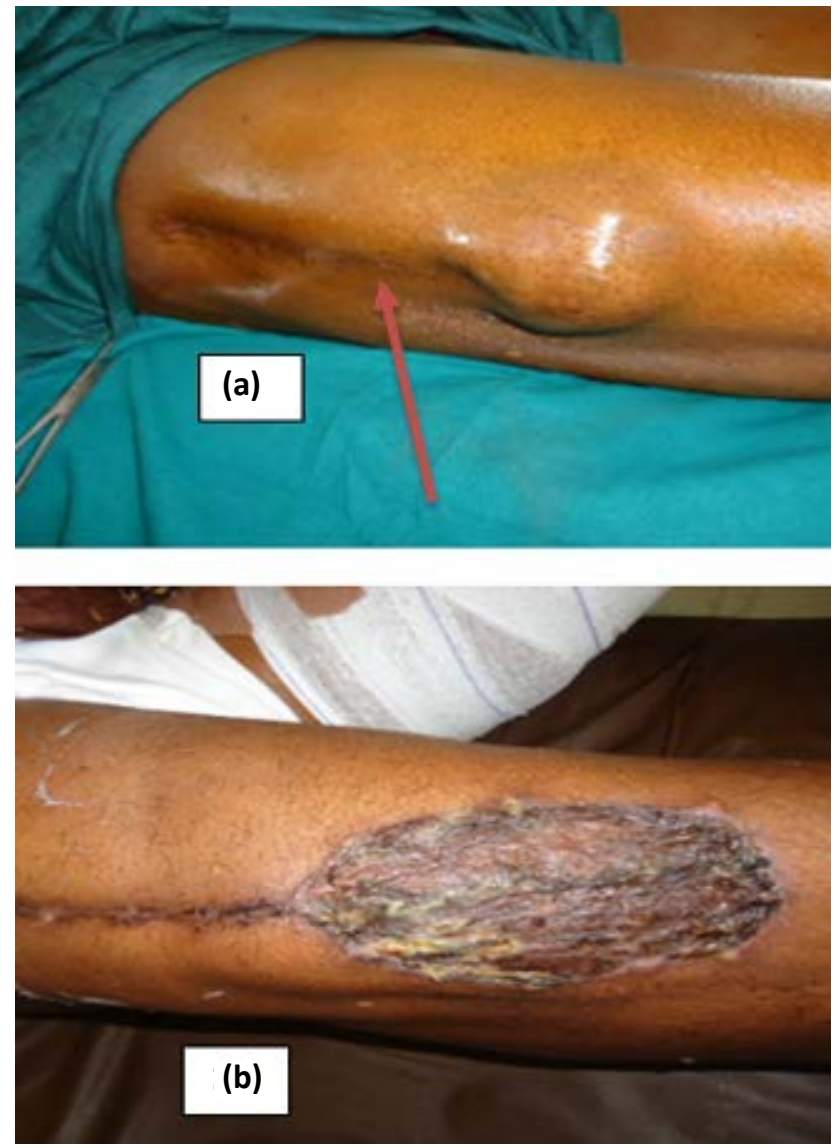

Figure 1. (a) pre-operative image with arrow pointing to old scar; (b) post-operative image.
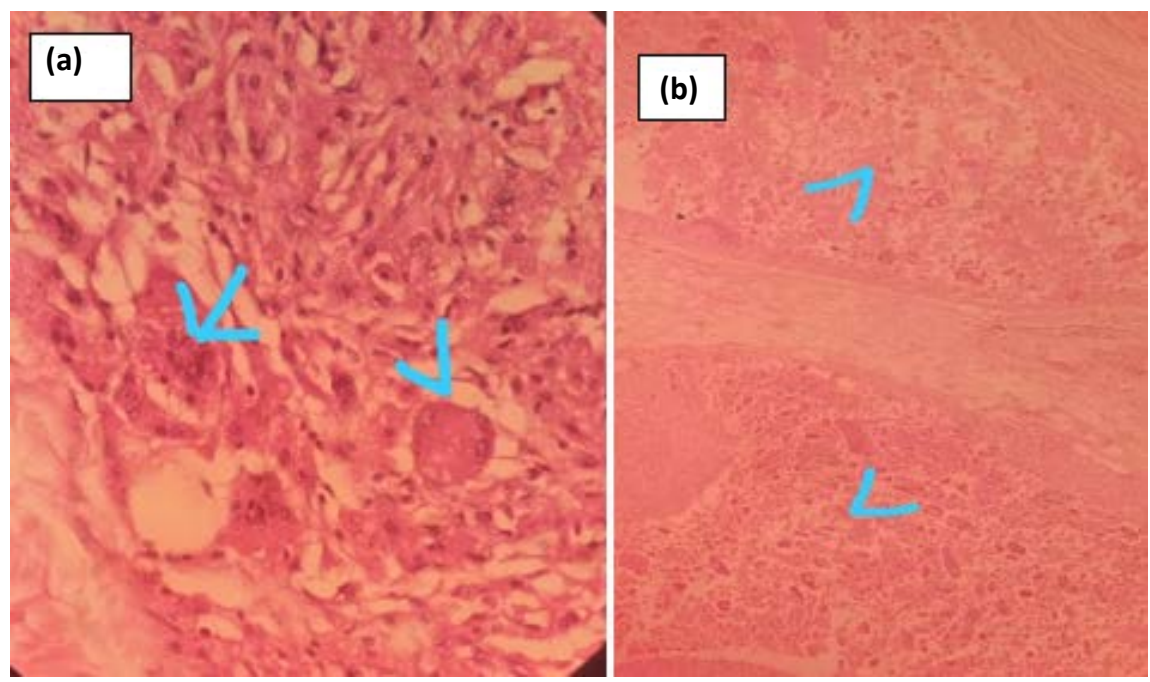

Figure 2. (a) Photomicrograph showing foreign body giant cells (arrow and arrow head) within a granuloma, (b) Photomicrograph showing pseudo cystic cavities (two arrow heads) containing insipated secretion.

HISTOLOGY foreign body type granulomatous reaction.

The patient was counseled and informed consent obtained for this case report. 


\section{Discussion}

The Lateral thigh fascia lata is a veritable source of autologous tissue for a wide variety of surgical procedures including interpositional arthroplasty for joint ankylosis, sling procedures for intrinsic sphincter deficiencies in women and ptosis correction, as well as a proven substitute tissue for the repair of heart valves, urethra, nasal septum and dura defects [2] [5] [6]. Complications following harvest of fascia lata from the lateral thigh are usually experienced in the early post operative period and comprise of haematoma and seroma formation, post-operative pain with attendant limping and wound complications such as infections and dehiscence. Careful and adequate attention to intraoperative techniques and post operative care usually limit the occurrence and progression of these events, hence most resolve or become inconsequential within six months of the surgical procedure as evident by the clinical studies of Giovannetti et al. [3]. Lateral cutaneous nerve hypoaesthesia and muscle prolapse have been reported by some authors as the presenting long term complications [3]. However, in the patients reviewed by Wheatcroft, Vardy and Tyers whose fascia lata graft size was not larger than $1.5 \times 15 \mathrm{~cm}$, the only long term concern was the scar cosmetic appearance [2].

Our index patient presented 48 yrs after the lateral thigh fascia lata harvest for interpositional arthroplasty with a donor site mass. Even though the only other positive tell-tale symptom is the progressively increasing size of the mass, the clinical diagnosis of soft tissue sarcoma was uppermost considering the age of the patient, site of tumour and history of previous surgical insult [4]. Histopathological confirmation of foreign body granulomatous reaction resolved the diagnostic dilemma as CT scan and MRI could not be done due to financial issues. These imaging studies have been reported to be complementary in the diagnosis of foreign body granuloma with CT scan having the advantage for radiopaque materials and MRI better deployed for radioluscent ones [7] [8]. However, if foreign body is not identifiable, the gadolinium enhanced surrounding inflammatory lesion is easily mistaken for soft tissue tumour on MRI [4] [7].

Foreign body granulomas are mainly grouped into gossipiboma from retained surgical sponge and foreign body induced granulations such as those caused by wooden splinters, a glass piece, bone fragments, sutures, silica etc. The history of surgical operation is obvious in our patient and the long term interval of forty eight years between the surgical encounter and clinical presentation of this case made the clinical diagnosis enigmatic. This also predicted a confusing radiological appearance on CT scan and MRI [7]. This index patient rightly had surgical exploration of the mass and excision of the well encapsulated myxomatous tissue with part of the overlying skin and scar, and split thickness skin graft resurfacing of the residual cutaneous defect. Lack of pre-operative definitive diagnosis and a high suspicion of a sarcomatous lesion necessitated a more extensive resection of the mass since central degeneration observed equally occurs in soft tissue sarcoma, a close differential previously reported by several authors [4] [8] [9]. The 
observed histological appearance of numerous prominent granulomas involving the dermis with foreign body type multinucleated giant cells is consistent with observations of others in previous surgical sites or invasive procedures where foreign body granulomas have developed [9] [10].

Following a follow up period of 3 years, no evidence of recurrence was found. Although the offending substance that triggered the granulomatous reaction could not be ascertained, both absorbable [11] and non-absorbable (9) surgical materials have been previously implicated. However, the complete gross removal of the myxomatous material assured us of the clearance of the degenerating foreign body from the fascia lata donor site and therewith satisfactory relief to the patient.

\section{Conclusion}

Foreign body granuloma can occur anywhere in the body especially following a history of surgical procedure regardless of the time interval between the surgical procedure and the clinical presentation. It continues to be a close differential of soft tissue tumour arising in previous surgical sites.

\section{Conflicts of Interest}

The authors declare no conflicts of interest regarding the publication of this paper.

\section{References}

[1] Amir, A., Gatot, A., Zucker, G., Sagi, A. and Fliss, D.M. (2000) Harvesting Large Fascia Lata Sheaths: A Rational Approach. Skull Base Surgery, 10, 29-34.

[2] Wheatcroft, S.M., Vardy, S.M. and Tyers, A.G. (1997) Complications of Fascia Lata Harvesting for Ptosis Surgery. British Journal of Ophthalmology, 81, 581-583. https://doi.org/10.1136/bjo.81.7.581

[3] Giovannetti, F., Barbera, G., Priore, P., Pucci, R., Della Monaca, M. and Valentini, V. (2019) Fascia Lata Harvesting: The Donor Site Closure Morbidity. Journal of Craniofacial Surgery, 30, E303-E306. https://doi.org/10.1097/SCS.0000000000005223

[4] Nakamura, T., Kusuzaki, K., Matsubara, T., Matsumine, A. and Uchida, A. (2008) Foreign-Body Granulomas in the Trunk and Extremities May Simulate Malignant Soft-Tissue Tumors: Report of Three Cases. Acta Radiologica, 49, 80-83. https://doi.org/10.1080/02841850701675727

[5] Alemán, R.M. and Martínez, M.G. (2012) Lateral Thigh Fascia Lata as Interpositional Graft for Temporomandibular Joint Ankylosis. Journal of Maxillofacial and Oral Surgery, 11, 354-357. https://doi.org/10.1007/s12663-011-0304-Z

[6] Lee, D., Alhalabi, F. and Zimmern, P.E. (2017) Long-Term Outcomes of Autologous Fascia Lata Sling for Stress Incontinence Secondary to Intrinsic Sphincter Deficiency in Women. Urological Science, 28, 135-138. https://doi.org/10.1016/j.urols.2017.03.002

[7] Ando, A., Hatori, M., Hagiwara, Y., Isefuku, S. and Itoi, E. (2009) Imaging Features of Foreign Body Granuloma in the Lower Extremities Mimicking a Soft Tissue Neoplasm. Upsala Journal of Medical Sciences, 114, 46-51. 
https://doi.org/10.1080/03009730802602455

[8] Sandhu, G.S., Parkash, M. and Bhatia, V. (2019) Pseudotumor of Hand Foreign Body Granuloma. Indian Journal of Musculoskeletal Radiology, 1, 72-74.

https://doi.org/10.25259/I]MSR 22019

[9] Morimoto, M., Takahashi, M., Sato, N., Nishisho, T., Kagawa, S., Kudo, E., et al. (2012) Expansively Hemorrhagic Foreign Body Granuloma at the Pelvis Caused by Microscopic Materials. Open Journal of Orthopedics, 2, 1-5. https://doi.org/10.4236/ojo.2012.21001

[10] Thway, K., Strauss, D.C., Smith, M.J. and Fisher, C. (2015) Foreign Body Granulomas Induced by Intramuscular Leuprorelin Acetate Injection for Prostate Cancer: Clinical Mimics of Soft Tissue Sarcoma. Case Reports in Oncological Medicine, 2015, Article ID: 947040. https://doi.org/10.1155/2015/947040

[11] Luo, J., Mao, Y., Cai, S., Shen, X., Chen, S. and Xie, L. (2014) Post-Nephrectomy Foreign-Body Granuloma in the Retroperitoneum Mimicking Lymph Node Metastasis of Renal Cell Cancer. OncoTargets and Therapy, 7, 2137-2141.

https://doi.org/10.2147/OTT.S70705 